\title{
Perfil de la madre adolescente primípara, en el servicio de puerperio de un hospital nacional de Lima
}

\author{
Sheyla Aguilar-Alarcón ${ }^{1, a}$, Shirley Zaguma-Serna ${ }^{2, a}$, Miriam García-Heredia $^{2, a}$, Raquel Meléndez-De la Cruz
}

\section{RESUMEN}

Objetivo: determinar el perfil de la adolescente puérpera atendida en el servicio de puerperio de un hospital nacional de Lima, en relación a las condiciones personales, condiciones de la salud sexual y reproductiva, condiciones familiares, sociales y económicas; y necesidades relacionadas al cuidado de su hijo y su autocuidado. Material y métodos: estudio descriptivo de corte transversal. La muestra estuvo conformada por 57 adolescentes puérperas primíparas que cumplieron los criterios de selección. La recolección de datos se realizó con un cuestionario elaborado por las investigadoras, el cual fue sometido a pruebas de validez y confiabilidad Resultados: la adolescente puérpera se encuentra entre 18 y 19 años de edad, con secundaria incompleta, y desean seguir estudiando. El inicio de las relaciones sexuales se da en la adolescencia tardía de 15 a 19 años, no usaron métodos anticonceptivos antes del embarazo. El $19,3 \%$ presentó complicaciones y el 12,3\% presentó un aborto previo. Son amas de casa en su mayoría, viven con sus parejas, confían más en la pareja, antecedente de ser hija de madre adolescente. Tienen diversas necesidades sobre el cuidado del recién nacido y su autocuidado. Conclusiones: Según el perfil identificado existen condiciones personales, familiares y sociales, siendo fundamental el soporte familiar y de enfermería para el afrontamiento de esta nueva etapa, por lo que se debe tener en cuenta las necesidades para su autocuidado y el de su hijo.

Palabras claves: embarazo en la adolescencia, salud reproductiva, periodo de postparto. (Fuente DesCS Bireme)

\begin{abstract}
Objective: The aim of the project was to determine the postpartum adolescent mother' profile treated at a postpartum unit in Lima, in relation to personal, sexual and reproductive health, family, social and economic conditions; and needs related to the child care and the mother's self care. Material and methods: A descriptive and cross-sectional research was conducted. A systematic sample of 57 postpartum primipara adolescents who fulfilled the selection criteria. A questionnaire created by the researchers that has 44 questions was divided into four sections. An expert review was conducted to assess the credibility and validity of the instrument. Statistical analysis of the quantitative data was conducted using frequency and percentage. Results: postpartum adolescents are between the age of 18 and 19 with incomplete secondary education, and want to continue studying. The initiation of sexual relations appears in late adolescents from 15 to 19 years old, did not use contraceptives before pregnancy, $19.3 \%$ presented complications and $12.3 \%$ had an abortion. Most of them are housewives who live with, trust their partners and have a history of being a teenage mother'daughter. The important basic educational need considered is to receive education about newborn care and less important is the mother's self care. Conclusions: According to the identified profile, there are personal, family and social conditions, being essential family and nursing support to cope with this new stage, therefore, is important to consider child and mother's care.
\end{abstract}

Key words: teenage pregnancy, reproductive health, postpartum period.

\footnotetext{
Ministerio de Salud. Lima, Perú.

Universidad Cesar Vallejo. Lima, Perú

Facultad de Enfermería, Universidad Peruana Cayetano Heredia.Lima- Perú

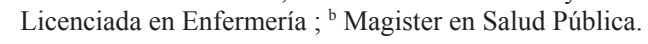




\section{INTRODUCCIÓN}

La palabra adolescencia viene del verbo latino "adolescere", que significa crecer o llegar a la madurez biológicasexual, psicológica y social. Una de las posturas considera a la adolescencia como una etapa de vida construida socialmente, otra, es una etapa de vida comprendida entre los 10 a 19 años, período de vida considerada para la Organización Mundial de la Salud (OMS) donde el individuo adquiere madurez reproductiva, transitan los patrones biológicos y psicológicos de la niñez a la adultez. La adolescencia es además, una etapa de múltiples posibilidades en la que se adoptan y consolidan hábitos $\mathrm{y}$ conductas que pueden afectar positiva o negativamente su salud para toda la vida (1-3).

En el mundo actual, el embarazo en la adolescencia en un problema de salud pública, a nivel mundial representa del 15 a $25 \%$ del total de embarazos, a nivel de América Latina y el Caribe se ha incrementado significativamente (4). El embarazo temprano contribuye a elevar la morbilidad y mortalidad materna, perinatal e infantil, y por sus consecuencias psicosociales, menos analizada en torno a los condicionantes socioculturales. Por lo que pese a los grandes esfuerzos desplegados, aún persisten las cifras de madres adolescentes.

La madre adolescente primípara presenta constantes cambios físicos, psicológicos y sociales, que se encuentran relacionadas con su propia etapa de desarrollo y que se caracterizan por aspectos vinculados a un proceso de madurez que aún no se encuentra completo tanto física y emocionalmente, afrontando una maternidad precoz, lo que hace que exista escaso conocimiento acerca de los cuidados tanto a su niño/a así como su propio cuidado.

El puerperio es el período de ajuste posterior al parto, durante el cual los cambios anatómicos y funcionales retornan a su estado normal no gestacional. Es probable que la adolescente se encuentre en un proceso manifestando un aumento de las tensiones fisiológicas y por la gran transición psicológica que experimenta, así como es más vulnerable debido al agotamiento de las energías, a la fatiga propia del fin del embarazo y del parto, el trauma tisular, pérdida de sangre y la anemia, pudiendo ocurrir complicaciones (5).

El profesional enfermero establece estrategias preventivas que contribuyan a que esta experiencia de ser madre adolescente no se convierta en el detenimiento del proyecto de vida, sino que encuentre el apoyo profesional oportuno para la adaptación y afrontamiento. En tal sentido, la información y orientación que reciban en materia de salud respecto a la promoción y prevención son de primordial importancia para su desarrollo integral y el de su hijo. La atención en salud de la puérpera se centra en el aspecto biologicista, aspecto importante; pero que debe ser integral, por todo lo que significa para una adolescente ser madre.

El estudio aborda el perfil de las madres adolescentes puérpera en relación a la caracterización de las condiciones personales; aspecto sexual y reproductivo, condiciones familiares y socioeconómicas y sobre las necesidades de cuidado percibidas para cuidar de su recién nacido, así como de su autocuidado, con el propósito de facilitar y fundamentar el cuidado enfermero integral a las madres adolescentes en el servicio de puerperio en el marco del derecho a una salud sexual y reproductiva como posibilidad de tener relaciones sexuales enriquecedoras sin coerción y sin temor de infección, de poder regular la fecundidad sin riesgos de efectos secundarios desagradables o peligrosos, de tener un embarazo y parto seguro, y de criar hijos saludables (6).

\section{MATERIAL Y MÉTODOS}

Estudio descriptivo de corte transversal, se realizó en el año 2013, en el Hospital Nacional Arzobispo Loayza, establecimiento de salud de nivel III y de referencia nacional, cuenta con el servicio de puerperio. En el estudio participaron 57 madres adolescentes, que cumplieron con los criterios de inclusión: puérperas primíparas entre 10 y 19 años de edad, que aceptaran participar voluntariamente en el estudio previa firma del consentimiento a mayores de 18 y los padres o apoderados y asentimiento informado en los adolescentes. Como criterio de exclusión se consideró que tuvieran alguna dificultad para responder al instrumento del estudio.

La técnica para la recopilación de datos fue la encuesta, se utilizó como instrumento el cuestionario elaborado por las investigadoras para los fines de este estudio, que fue sometido a pruebas de validez y confiabilidad. Después de haber obtenido la autorización del Comité Institucional de Ética de la UPCH y del Comité de ética del Hospital Nacional Arzobispo Loayza, se coordinó con la enfermera jefe del Servicio de Puerperio para informarle las características de la investigación, la forma de recojo de información y establecer el cronograma de la aplicación del instrumento.

Se seleccionó a las madres que cumplieron los criterios de selección, se informó de manera individual sobre el propósito, objetivo, metodología y consideraciones éticas, se entregó la hoja de consentimiento informado a las madres adolescentes mayores de 18 años; y se entregó el 
asentimiento informado a las menores y consentimiento informado a sus padres o apoderados, la aplicación del instrumento fue de 30 minutos de duración aproximadamente.

Los datos obtenidos se codificaron, previo control de calidad del llenado del cuestionario, y el procesamiento de los datos se realizó mediante el software estadístico SPSS versión 17. Los hallazgos se presentaron considerando la distribución de frecuencias y porcentajes.

\section{RESULTADOS}

Tabla 1. Condiciones personales de la adolescente atendida en el servicio de puerperio de un Hospital Nacional de Lima

\begin{tabular}{|l|c|c|}
\hline DEMOGRÁFICOS & $\mathrm{n}$ & $\mathbf{( \% )}$ \\
\hline Edad & & \\
\hline $15-17$ & 17 & 29,8 \\
\hline $18-19$ & 40 & 70,2 \\
\hline Lugar procedencia & & \\
\hline DISA V Lima Ciudad & 52 & 91,2 \\
\hline DIRESA Callao & 5 & 8,8 \\
\hline Estado civil & & \\
\hline Conviviente & 41 & 71,9 \\
\hline Soltera & 14 & 24,6 \\
\hline Casada & 2 & 3,5 \\
\hline ESTUDIOS & & \\
\hline Grado escolaridad & & \\
\hline Primaria & 3 & 5,3 \\
\hline Secundaria & 47 & 82,5 \\
\hline Superior técnico incompleto & 7 & 12,3 \\
\hline Desea estudiar después & & \\
\hline Sí & 48 & 84,2 \\
\hline No & 9 & 15,8 \\
\hline
\end{tabular}

En la tabla se observa que el $70,2 \%$ de las adolescentes tuvieron entre 18 y 19 años de edad; el lugar de procedencia del 91,2\% fue de Lima Ciudad, el 71.9\% reportó ser conviviente y en un $24,5 \%$ madre soltera; el $82,5 \%$ cursó secundaria y el $84,2 \%$ desea seguir estudiando después.
Tabla 2. Condiciones del comportamiento sexual y reproductivo de la adolescente atendida en el servicio de puerperio de un Hospital Nacional de Lima.

\begin{tabular}{|c|c|c|}
\hline ACTIVIDAD SEXUAL & $\mathrm{N}$ & $(\%)$ \\
\hline \multicolumn{3}{|c|}{ Edad de inicio de relaciones sexuales } \\
\hline $10-14$ años & 7 & 12,3 \\
\hline $15-19$ años & 50 & 87,7 \\
\hline \multicolumn{3}{|l|}{ Número de compañeros sexuales } \\
\hline 1 & 39 & 68,4 \\
\hline 2 & 11 & 19,3 \\
\hline 3 a más & 7 & 12,3 \\
\hline \multicolumn{3}{|c|}{ SITUACIÓN EN QUE SE PRESENTÓ EL EMBARAZO } \\
\hline Relación sexual consentida & 44 & 77,2 \\
\hline Otro & 8 & 14,0 \\
\hline Alcohol & 4 & 7,0 \\
\hline Drogas & 1 & 1,8 \\
\hline \multicolumn{3}{|c|}{ RAZONES PARA QUEDAR EMBARAZADA } \\
\hline Por amor & 19 & 33,3 \\
\hline Irse a vivir con su pareja & 15 & 26,3 \\
\hline Tener un motivo para vivir & 8 & 14,0 \\
\hline Curiosidad, escasa información & 5 & 8,8 \\
\hline Salir de casa & 4 & 7,0 \\
\hline No usar anticonceptivo & 4 & 7,0 \\
\hline Rebelión contra los padres & 2 & 3,5 \\
\hline \multicolumn{3}{|l|}{ PLANIFICACIÓN FAMILIAR } \\
\hline \multicolumn{3}{|l|}{ Usó métodos anticonceptivos } \\
\hline Ninguno & 27 & 47,4 \\
\hline Método del ritmo & 16 & 28,1 \\
\hline Preservativo & 11 & 19,3 \\
\hline Hormonal & 3 & 5,3 \\
\hline \multicolumn{3}{|l|}{ Método anticonceptivo a seguir } \\
\hline Hormonal & 19 & 33,3 \\
\hline Método del ritmo & 18 & 31,6 \\
\hline Preservativo & 11 & 19,3 \\
\hline Ninguno & 9 & 15,8 \\
\hline \multicolumn{3}{|l|}{ GINECOLÓGICOS } \\
\hline \multicolumn{3}{|l|}{ Tipo de parto } \\
\hline Eutócico & 47 & 82,5 \\
\hline Distócico & 10 & 17,5 \\
\hline \multicolumn{3}{|c|}{ COMPLICACIONES DEL PUERPERIO } \\
\hline Ninguna & 46 & 80,7 \\
\hline Alguna complicación & 11 & 19,3 \\
\hline \multicolumn{3}{|l|}{ Número de abortos } \\
\hline Ninguno & 50 & 87,7 \\
\hline Alguno & 7 & 12,3 \\
\hline
\end{tabular}

El 87,7\% de adolescentes puérperas inició sus relaciones sexuales entre los 15 y 19 años de edad; el 68,4\% sólo un compañero sexual; en cuanto a la situación en que se dio el embarazo el $77,2 \%$ fue por relaciones sexuales consentidas, y el 7,0\% fue bajo los efectos del alcohol; con respecto a las razones para quedar embarazada el 33,3\% dice que fue por amor, en cuanto al método anticonceptivo el 33,3\% de adolescentes refieren que usarían. El 82,5\% tuvo un tipo parto eutócico, el 19,3\% presentó complicaciones y el $12,3 \%$ presentó un aborto. 
Tabla 3. Condiciones familiares, sociales y económicas de la adolescente atendida en el servicio de puerperio de un Hospital Nacional de Lima

\begin{tabular}{|c|c|c|}
\hline FAMILIAR & $\mathrm{n}$ & $(\%)$ \\
\hline \multicolumn{3}{|l|}{ Con quien vive } \\
\hline Pareja o esposo & 45 & 78,9 \\
\hline Padres & 11 & 19,3 \\
\hline Abuelos & 1 & 1,8 \\
\hline \multicolumn{3}{|l|}{ Tipo de familia } \\
\hline Padres casados o juntos & 31 & 54,4 \\
\hline Padres divorciados o separados & 24 & 42,1 \\
\hline Madre o padre viudos & 2 & 3,5 \\
\hline \multicolumn{3}{|l|}{ Hay más confianza } \\
\hline Pareja & 30 & 52,6 \\
\hline Madre & 19 & 33,3 \\
\hline Padre & 8 & 14,0 \\
\hline \multicolumn{3}{|l|}{ Relación con la madre } \\
\hline Muy buena & 9 & 15,8 \\
\hline Buena & 41 & 71,9 \\
\hline Regular & 5 & 8,8 \\
\hline Mala & 1 & 1,8 \\
\hline Muy mala & 1 & 1,8 \\
\hline \multicolumn{3}{|l|}{ Relación con el padre } \\
\hline Muy buena & 7 & 12,3 \\
\hline Buena & 32 & 56,1 \\
\hline Regular & 11 & 19,3 \\
\hline Mala & 4 & 7,0 \\
\hline Muy mala & 3 & 5,3 \\
\hline \multicolumn{3}{|l|}{ Maltrato de la familia } \\
\hline Sí & 13 & 22,8 \\
\hline No & 44 & 77,2 \\
\hline \multicolumn{3}{|l|}{ Violencia de la pareja } \\
\hline Sí & 6 & 10,5 \\
\hline No & 51 & 89,5 \\
\hline \multicolumn{3}{|l|}{ SOCIAL } \\
\hline \multicolumn{3}{|c|}{ Grado de escolaridad de la madre } \\
\hline Analfabeta & 6 & 10,5 \\
\hline Primaria & 14 & 24,6 \\
\hline Secundaria & 28 & 49,1 \\
\hline Superior técnico & 7 & 12,3 \\
\hline Superior universitario & 2 & 3,5 \\
\hline \multicolumn{3}{|l|}{ Grado de escolaridad del padre } \\
\hline Analfabeto & 4 & 7,0 \\
\hline Primaria & 12 & 21,1 \\
\hline Secundaria & 29 & 50,9 \\
\hline Superior técnico & 9 & 15,8 \\
\hline Superior universitario & 3 & 5,3 \\
\hline \multicolumn{3}{|l|}{ Edad de la pareja } \\
\hline $17-19$ años & 25 & 43,9 \\
\hline $20-23$ años & 32 & 56,1 \\
\hline \multicolumn{3}{|c|}{ Edad de su madre en el primer hijo } \\
\hline $15-19$ años & 46 & 80,7 \\
\hline $20-23$ años & 11 & 19,3 \\
\hline \multicolumn{3}{|l|}{ ECONÓMICA } \\
\hline \multicolumn{3}{|l|}{ Ocupación actual } \\
\hline Ama de casa & 42 & 73,7 \\
\hline trabajo dependiente & 11 & 19,3 \\
\hline trabajo independiente & 2 & 3,5 \\
\hline Estudiante & 1 & 1,8 \\
\hline estudia y trabaja & 1 & 1,8 \\
\hline \multicolumn{3}{|l|}{ Sustento económico actual } \\
\hline Pareja & 53 & 93,0 \\
\hline Padres/familia & 4 & 7,0 \\
\hline
\end{tabular}


La tabla muestra los aspectos familiares y socio- económicos, donde el 78,9\% vive con su pareja o esposo; los padres de las encuestadas son casados o viven juntos en un $54,4 \%$; un $80.7 \%$ de las madres de las adolescentes tuvo su primer hijo entre los 15 y 19 años, el 22,8\% re- cibió maltrato por parte de la familia y el 10,5\% recibió violencia por parte de la pareja. E1 56,1\% de las parejas sexuales tiene entre 20 y 23 años de edad; en cuanto a la ocupación actual el 73,7\% son amas de casa; el sustento económico actual del 93,0\% es su pareja.

Tabla 4. Necesidades educativas básicas de la adolescente atendida en el servicio de puerperio de un Hospital Nacional de Lima

\begin{tabular}{|c|c|c|c|}
\hline Necesidades educativas de cuidado de su hijo & Importante & Poco importante & No es importante \\
\hline & $(\%)$ & $(\%)$ & $(\%)$ \\
\hline Frecuencia de la alimentación al pecho & 64,9 & 19,3 & 15,8 \\
\hline Lactancia materna como alimento exclusivo & 56,1 & 19,3 & 24,6 \\
\hline Posiciones para amamantar & 56,1 & 28,1 & 15,8 \\
\hline Precauciones para bañar al bebé & 66,7 & 19,3 & 14,0 \\
\hline Cuidados del cordón umbilical & 64,9 & 21,1 & 14,0 \\
\hline Recomendaciones para vestir al bebé & 54,4 & 31,6 & 14,0 \\
\hline Formas de expresar afecto & 66,7 & 28,1 & 5,3 \\
\hline Posiciones para dormir al bebé & 63,2 & 24,6 & 12,3 \\
\hline Estimulación temprana & 77,2 & 19,3 & 3,5 \\
\hline Vacunas & 54,4 & 29,8 & 15,8 \\
\hline Necesidades educativas de autocuidado & $\begin{array}{c}\text { Importante } \\
(\%) \\
\end{array}$ & $\begin{array}{c}\text { Poco importante } \\
(\%) \\
\end{array}$ & $\begin{array}{c}\text { No es importante } \\
(\%) \\
\end{array}$ \\
\hline Alimentación & 59,6 & 31,6 & 8,8 \\
\hline Higiene & 61,4 & 28,1 & 10,5 \\
\hline Actividad física & 38,6 & 49,1 & 12,3 \\
\hline Planificación familiar & 54,4 & 17,5 & 28,1 \\
\hline Descanso & 29,8 & 61,4 & 8,8 \\
\hline Cuidado de mamas & 66,7 & 21,1 & 12,3 \\
\hline
\end{tabular}

Las adolescentes puérperas consideran importante en un $77,2 \%$ conocersobre estimulación temprana, un $66.7 \%$ precauciones para bañar al bebé e igual porcentaje formas de expresar afecto al recién nacido, 64.9\% la frecuencia de la alimentación al pecho, en igual porcentaje cuidados del cordón umbilical, 63,2\%; Sobre su autocuidado, consideran importante en un $66.7 \%$ el cuidado de mamas, $61,4 \%$ sobre higiene, 59,6\% sobre alimentación saludable, y $54,4 \%$ sobre planificación familiar.

\section{DISCUSIÓN}

El estudio fue realizado en el servicio de puerperio del Hospital Nacional Arzobispo Loayza, donde en promedio más del 14\% de usuarias que acuden al servicio son adolescentes primíparas.

Los hallazgos sobre las condiciones personales, mostraron que la edad de las adolescentes puérperas está entre 15 y 19 años, coinciden con el resultado presentado por ENDES 2013, donde un 2,0\% inicia la procreación a los 15 años, llegando hasta el 30,2\% en las de 19 años. En el Perú el porcentaje de quienes son madres o están embarazadas es $13.9 \%$, de éstas el $10.5 \%$ son madres y el 3,5\% están gestando por primera vez. El mayor número de adolescentes puérperas proceden de la jurisdicción de la DISA V Lima Ciudad, los resultados son coincidentes con la ENDES 2013, en donde hay un mayor número de adolescentes madres en Lima metropolitana en los últimos años (7).

Los resultados demostraron que el 71,9\% tiene estado civil conviviente y el $24,6 \%$ son madres solteras, lo cual resulta riesgoso ya que la pareja comprometida en matrimonio otorga estabilidad emocional, afectiva y económica. En la adolescencia las parejas adolescentes se caracterizan por ser de menor duración y más inestables, lo que suele magnificarse por la presencia del primer hijo concebido antes del matrimonio, debido a que uno de los aspectos que caracteriza a la maternidad adolescente es que se realiza en un contexto donde las uniones legales son infrecuentes, haciendo que la madre afronte su maternidad sin el apoyo del padre de su hijo, desprotegida, lo cual las vuelve vulnerable.

En cuanto al grado de escolaridad de las adolescentes puérperas, los resultados demostraron que el 82,5\% estudiaron secundaria incompleta, la mayoría de las adolescentes puérperas al quedar embarazadas dejan sus estudios por la gestación y más adelante para cuidar y atender a sus hijos, 
esta situación hace que más adelante por su necesidad económica empezará a trabajar sin ser bien remunerada debido al bajo nivel educativo, lo cual por consecuencia reproduce la pobreza como resalta Mendoza (8). La mayoría de embarazos se dan a edades en que la adolescente se encuentra estudiando y con expectativas para seguir alguna carrera o planes de vida que difieren a lo que experimenta al encontrarse afrontando la maternidad, razón por la cual el seguir estudiando dependerá del esfuerzo de ellas, y que cuenten con el apoyo en el cuidado de su hijo; tanto por la pareja como por la familia.

Respecto a las condiciones de la conducta sexual y reproductiva, se halló que la edad de inicio de las relaciones sexuales predomina entre los 15 y 19 años con un $87,8 \%$, lo que coincide con ENDES continua 2011 donde el 63\% inician relaciones sexuales antes de los 20 años, siendo la edad media de inicio sexual a los 19 años en zonas urbanas. Uno de los condicionantes sería la influencia de la presión de pares y la inmensa cantidad de estímulos sexuales difundidos por medios masivos de comunicación, así como el internet y redes sociales, de esta manera ejercen su sexualidad en pos del deseo y búsqueda del placer, como parte de su desarrollo, pero sin el autocuidado que se requiere (9).

Dentro de las situaciones en que se presentó el embarazo los resultados denotan que el $77,2 \%$ fueron por relaciones consentidas, la adolescente deseaba embarazarse para vivir en pareja y para salirse de su casa caracterizado por un entorno de violencia según sostiene la ENDES 2013 (7), y estudios de índole psicológico encuentran que la maternidad se da en búsqueda de identidad (10).En un 7\% la situación se dio bajo los efectos del alcohol, existe una cultura producto de la imitación y la presión de pares, asimismo la permisividad por parte de los padres, en lo referente a la vida social de sus hijos fuera del hogar, debido a la disminución de reglas en el hogar. Todas estas condiciones favorecen una relación sexual casual, existiendo facilidades con las que cuenta el medio capitalino en la que abundan espacios de diversión asociados al consumo de alcohol, estimulante que provoca permisibilidad y toma de decisiones poco razonadas, es decir que se acepta tener relaciones sexuales sin protección. MINSA a través de un Plan Nacional de Lucha Contra el Consumo Nocivo de Alcohol, precisa que el consumo cada vez a edades más tempranas podría repercutir en las facultades para la toma de decisión de la adolescente frente a una propuesta de relación sexual (11).

Es importante que la adolescente reciba consejería para decidir sobre el método anticonceptivo un $33,3 \%$ dijo que usaría un método hormonal, seguido al 31,6\% que usaría el método del ritmo y el 15,8\% no desea seguir ningún método de planificación familiar. Un 47,4\% de las adolescentes puérperas no usó ningún método anticonceptivo antes del embarazo y el 28,1\% usó el método del ritmo. Existen muchos mitos para que los adolescentes no accedan a un método anticonceptivo, que han sido reportados en varios estudios (12). Todo lo cual debería tomarse en cuenta en la información, educación, consejería, y servicios en materia de salud sexual y reproductiva que fomente una sexualidad sana y responsable.

El 17,5\% presentó un parto distócico, en el caso de las adolescentes es necesario llevar un buen control de su embarazo, debido a que en su condición de adolescente su cuerpo aún no está físicamente preparado para alumbrar a un bebe, ya que no se ha completado el desarrollo óseo y no se ha alcanzado la masa mineral máxima, así como la relativa inmadurez del canal del parto, pudiendo presentar complicaciones como parto distócico, parto prolongado, desproporción céfalo-pélvica, y formación de fistulas recto-vaginales, y en casos extremos pueden perder la vida(12).

El 19,3\% tuvo al menos una complicación en el puerperio lo que incluye infecciones, preeclampsia y hemorragia. Autores como Reeder y Koniak, mencionan que las hemorragias e infecciones son dos complicaciones y causas frecuentes de morbimortalidad en puérperas adolescentes (13).

El 54,4\% de los padres de las encuestadas son casados o viven juntos y el $41,2 \%$ tuvo padres divorciados o separados. El tener una madre sola (viuda o separada) incrementa la probabilidad de iniciar tempranamente las relaciones sexuales (14).

Un $71,9 \%$ y $56,1 \%$ tienen una buena relación con la madre y el padre respectivamente, al respecto Diego y Huarcaya (15) señalan que la relación familiar tiende a entrar en conflicto, en el comienzo de la adolescencia y disminuye hacia el final de esta. Según estudios realizados la relación con los padres es mala. Siendo escaso el apoyo familiar, ayuda económica y/o emocional que la adolescente y su hijo reciben de parte de sus padres $\mathrm{u}$ otro familiar, considerando que por encontrarse el adolescente con un embarazo y una maternidad inesperada para ella y los padres, pueden encontrar rechazo y verse desprotegida o desprovista de apoyo, en el caso de los padres a pesar de no estar de acuerdo con esta situación inesperada, proporcionan ayuda económica para ella y su hijo, pero posiblemente les sea más difícil brindarle apoyo emocional, porque también están pasando por un proceso de adaptación. 
Los resultados demostraron que las madres de las adolescentes puérperas tuvieron su primer hijo entre 15 y 19 años; es decir, también durante su adolescencia. Existe una transmisión intergeneracional de la maternidad adolescente, la que es posible que cree una serie de desventajas en las hijas de estas mujeres, reproduciendo la pobreza, ya que posiblemente sus hijas al ser adolescentes puérperas también alcancen menor escolaridad al no terminar sus estudios para dedicarse al cuidado de sus hijos, los valores familiares y de su entorno cultural-social contribuyen a la conducta sexual.

Con respecto a la violencia familiar los resultados demostraron que un porcentaje importante de adolescentes puérperas encuestadas $22,8 \%$ recibió maltrato por parte de la familia y el 10,5\% recibió violencia por parte de la pareja. La razón más comúnmente relacionada con el alto riesgo de violencia familiar hacia la madre adolescente, es el aumento de estrés que siente la familia de la adolescente o la pareja con respecto a la llegada inminente del nuevo ser, y este estrés se manifiesta como una frustración dirigida contra la madre y su niño. La violencia en una adolescente puérpera puede tener alcances mortales para la madre y el bebé, generando lesiones en donde el impacto mayor es en la salud mental de la mujer y en el niño; desarrollando un cuadro de estrés en la adolescente puérpera permanentemente asociado con depresión, baja autoestima, aislamiento social, entre otros (16).

E1 73,7\% de madres adolescentes no trabaja ni estudia y se dedicará al cuidado de su hijo y su hogar, no cuenta con ayuda familiar para el cuidado de sus hijos; por lo tanto, se dedican a ser amas de casa, y no participan en el mercado laboral, no porque no deseen trabajar sino porque carecen de formación laboral óptima que les permita insertarse en un mercado de trabajo muy competitivo.

En cuanto al sustento económico actual de las adolescentes puérperas encuestadas, los resultados demostraron que el 93,0\% está a cargo de su pareja y en el 7,0\% a cargo de los padres. Estos resultados podrían deberse al nuevo rol que asumen los padres adolescentes por las responsabilidades parentales, donde es el varón el que tiene que asumir la manutención de la familia, sin embargo el bajo nivel de formación, y la habitualmente escasa o nula experiencia laboral, hacen difícil que encuentren un trabajo óptimo; como consecuencia viven con reducidos ingresos. En algunos casos, la situación económica es tan precaria que pasan a depender de sus padres o de las familias de sus parejas, lo que representa una mayor carga para el grupo familiar $(17,18)$.

En relación a las condiciones de necesidades de apren- dizaje, según lo hallado, las adolescentes puérperas desconocen sobre los cuidados del recién nacido y sobre sus propios cuidados, pero si consideran importante recibir educación, permitiendo deducir que las adolescentes puérperas tengan dificultades para asumir el cuidado de su hijo, teniendo temor, pocas habilidades en el cuidado de un recién nacido debido a su inexperiencia como madre, lo que genera la necesidad de conocer sobre el cuidado de su niño para evitar que se enferme como lo resalta Alonso y cols. (19). Las madres adolescentes presentan bajos niveles de conocimiento y escasas habilidades en el cuidado de sus hijos, como lo señala Cardozo (20).

En los servicios de puerperio se brinda mayor cuidado sobre los aspectos relacionados al cuerpo es decir a lo biologicista, el equipo de salud en especial el enfermero debe implementar en base a una actitud pedagógica la consejería y/o orientación a la madre adolescente en torno a sus necesidades sobre el cuidado del niño y su propio cuidado, como lo contempla Blossiers (21).

\section{REFERENCIAS BIBLIOGRAFICAS}

1. Documento técnico: análisis de situación de salud de las y los adolescentes. Ubicándolo y ubicándonos. Ministerio de Salud. Perú 2009

2. Luna C. Diagnóstico sobre Embarazo Adolescente en el Perú. UNFPA. 2008 [Citado 09 mar. 2012]. Disponible en:ftp://www.unfpa.org.pe/LACRO-Masculinidades/PLAN-PEA-VERSION-ABRIL-2011.pdf

3. Claros A, Adolescentes multigestas: aspectos socioculturales. Revista Pediátrica [Internet], 2006 [Citado el 02 feb. 2012]; 8(1). Disponible en: http://sisbib. unmsm.edu.pe/bvrevistas/pediatrica/v08_n1/pdf/a03. pdf

4. MINSA. Prevención del Embarazo en Adolescentes. Disponible en: Prevención del embarazo en adolescentes [Internet] 2014. [Citado 16 mar. 2014]. Disponible en: http://www.minsa.gob.pe/portada/ Especiales/2014/adolescentes/_cont.asp?pg=1

5. Valdez F. Prevalencia y factores de riesgo para complicaciones obstétricas en adolescentes. Rev Gineco Obstetra 2006; 69: 209-213

6. Chávez S. Guerreo R. Concepciones y percepciones sobre los derechos sexuales y reproductivos en la adolescencia. 2007 Lima: CARE Perú, CIES, PROMSEX. $89-151$.

7. Encuesta demográfica y de salud familiar ENDES [Internet]. 2013. Disponible en http://www.inei.gob. 
pe/media/MenuRecursivo/publicaciones_digitales/ Est/Lib1151/index.html

8. Mendoza W, Subiría G. El embarazo adolescente en el Perú: Situación actual e implicancias para las políticas públicas [Internet]. 2013 [Citado 19 feb. 2015]. Disponible en: http://www.unfpa.org.pe/Articulos/Articulos/MENDOZA-SUBIRIA-EmbarazoAdolescente-Peru.pdf

9. Contreras S, Martín D. El embarazo en la adolescencia. ¿Un problema social en Santa Clara, Villa Clara, Cuba?, en Contribuciones a las Ciencias Sociales [Internet]. 2011 [Citado 09 mar. 2012]. Disponible en: http://www.eumed.net/rev/cccss/12/crmc.pdf

10. Bustamante I. Significados del embarazo y la maternidad en la construcción de sus identidades en adolescentes de 15 a 19 años de sector socio-económico bajo, que residen en la ciudad de Lima. [Tesis para optar el Grado de Magíster en Salud Pública]. Lima: Universidad Peruana Cayetano Heredia; 2000

11. Plan nacional de lucha contra el consumo nocivo de alcohol. [Citado 26 jul. 2013]. Disponible en http://www.minsa.gob.pe/portada/especiales/2008/ amorycontrol/archivos/Plan_de_Comunicaciones.pdf

12. PROMSEX. Adolescencia y acceso a salud reproductiva y educación sexual ¿Qué puedo cambiar? [Internet]. 2011 [Citado 26 jul. 2012]. Disponible en http://www.unfpa.org.pe/publicaciones/publicacionesperu/PROMSEX-Adolescencia-Acceso-SaludrReproductiva.pdf

13. Reeder S, Martín L, Koniak D. Enfermería MaternoInfantil. 17a ed. México DF: Editorial Interamericana McGraw-Hill; 2000.

14. Flórez CE, Factores socioeconómicos y contextuales que determinan la actividad reproductiva de las adoles- centes en Colombia. Rev. Panam. Salud Pública. [Internet]. 2005 [Citado 15 may. 2012]; 18(6):388-402. Disponible en: http://www.scielosp.org/pdf/rpsp/ v18n6/28940.pdf]

15. Diego P, Huarcaya G. Factores familiares y reproductivos asociados al embarazo reincidente en adolescentes INMP. [Internet]. 2008 [Citado 13 abr. 2012] [Disponible en http://www.cybertesis.edu.pe/sisbib/2008/ diego_dp/pdf/diego_dp.pdf]

16. Salaverry O. Embarazo en adolescentes de Lima. [Internet]. 2011 [Citado 26 jul. 2013] [Disponible en: www.angelfire.com/pe/actualidadpsi/embarazo.html]

17. Cruz MC. Embarazo de adolescentes y comunicación familiar. Margen61 [Internet]. 2011. [Citado 09 mar. 2012]. Disponible en: http://www.margen.org/suscri/ margen61/marquez.pdf]

18. Embarazo en adolescentes: un problema culturalmente complejo [Internet]. 2009. Disponible en: http://www. who.int/bulletin/volumes/87/6/09-020609/es/index. html [citado: 8 de julio de 2012].

19. Alonso M, Fariñas T, Rodríguez B, Campo A, Díaz R. Intervención educativa sobre la atención a niños de madres adolescentes. Guanabacoa. 2008 [Citado 12 DIC. 2012] [Disponible en: http://scielo.isciii.es/scielo.php?pid=S1132-12962010000300012\&script $=$ sci $\operatorname{arttext\& tlng}=\mathrm{pt}]$

20. Cardozo J. Conocimientos de madres adolescentes sobre cuidados básicos al recién nacido. Bogotá 2008. [Citado 12 DIC. 2012] [Disponible en: http://www. javeriana.edu.co/biblos/tesis/enfermeria/tesis09.pdf]

21. Blossiers C. Interacción entre el personal de salud y las jóvenes embarazadas durante el control prenatal: un estudio cualitativo. Rev. Perú Med Exp Salud Pública. 2010; 27(3): 337-44.

Fecha de recibido: 12 de Octubre de2014

Fecha de aceptación: 26 de Noviembre de 2014. 